\title{
Methodenentwicklung zur numerischen Strömungsanalyse von Freispiegelströmungen bei Schaufelwasserrädern
}

\author{
Hans-Henning Schippke, Christian Seidel, Dieter Dinkler, Andreas Zilian
}

Der Ausbau der Wasserkraft gewinnt im Kontext der Energiewende weltweit an Bedeutung. Neben der Turbinentechnologie erweisen sich Wasserräder im Hinblick auf ihre hohe ökologische Verträglichkeit und ihr hohes ganzjähriges Arbeitsvermögen als besonders geeignet.

Das sich drehende Wasserrad, umgeben von Wasser und Luft, stellt mechanisch ein gekoppeltes DreiFeld-System bestehend aus einer Struktur und zwei Fluiden dar. Hinreichend genau ist es möglich, die Wasserradstruktur als Starrkörper zu beschreiben, während Luft und Wasser mit Hilfe der inkompressiblen Navier-Stokes Gleichungen gut modelliert werden können.

Durch Anwendung der Raum-Zeit-Finite-Elemente-Methode zur Diskretisierung der inkompressiblen Navier-Stokes Gleichungen wird die Grenzfläche zwischen dem Starrkörper und den Fluiden explizit beschrieben und stets automatisch korrekt erfasst. Die Beschreibung der freien Wasseroberfläche als Grenzfläche zwischen den beiden Fluiden erfolgt implizit mit Hilfe der Level-Set Methode.

Die Netzknoten der Fluid-Struktur-Grenzfläche verändern ihre Position infolge der Wasserraddrehung mit der Zeit, so dass ein Netzbewegungsalgorithmus notwendig ist. Die shear-slip mesh update method (SSMUM) ermöglicht als diskontinuierliches Netzbewegungsverfahren eine durchgehende Berechnung des Gesamtsystems, ohne dass das gesamte Gebiet neu vernetzt werden muss.

Anhand verschiedener Testbeispiele wird die Güte der entwickelten numerischen Methode im Hinblick auf Ihre Erhaltungseigenschaften untersucht. Besonderes Augenmerk wird auf die Lösungsübertragung am shear-slip layer sowie auf die Abbildung der implizit beschriebenen freien Wasseroberfläche gelegt. 